\title{
Tissue Type-Specific Bioenergetic Abnormalities in Adults with Major Depression
}

\author{
David G Harper*,', J Eric Jensen', Caitlin Ravichandran', Roy H Perlis ${ }^{2}$, Maurizio Fava ${ }^{2}$, Perry F Renshaw ${ }^{3}$ and \\ Dan V losifescu ${ }^{4}$
}

'Department of Psychiatry, McLean Hospital and Harvard Medical School, Belmont, MA, USA; '2Department of Psychiatry, Massachusetts General Hospital and Harvard Medical School, Boston, MA, USA; ${ }^{3}$ The Brain Institute, University of Utah, Salt Lake City, UT, USA; ${ }^{4}$ Departments of

Psychiatry and Neuroscience, Icahn School of Medicine at Mount Sinai, New York, NY, USA

\begin{abstract}
Brain bioenergetic abnormalities have been observed frequently in adults with major depressive disorder (MDD); however, results have been inconsistent regarding whether decreased or increased metabolism was observed. Phosphorus-3I magnetic resonance spectroscopy (3IP MRS) allows for the quantification of bioenergetic molecules, containing high-energy phosphates, over the whole brain as well as measuring the differences between gray matter and white matter. We recruited 50 subjects with a current diagnosis of MDD, not currently treated with psychotropic medication, between ages of 18 and 65 (mean \pm SD age: $43.4 \pm$ 13.6; 46\% female) and 30 healthy volunteers, matched for age and gender (39.0 \pm 12.5 years of age; $36.6 \%$ female). All subjects received a TI MP-FLASH scan for tissue segmentation followed by 3 IP MRS, chemical shift imaging scan with 84 voxels of data collected over the entire brain utilizing a dual-tuned, protonphosphorus coil to minimize subject movement. Phosphocreatine and inorganic phosphate (Pi) varied in opposite directions across gray matter and white matter when MDD subjects were compared with controls. This finding suggests alterations in high-energy phosphate metabolism and regulation of oxidative phosphorylation in MDD patients. In addition, within the MDD group, gray matter Pi, a regulator of oxidative phosphorylation, correlated positively with severity of depression. These data support a model that includes changes in brain bioenergetic function in subjects with major depression.

Neuropsychopharmacology (2017) 42, 876-885; doi:I0.1038/npp.2016.180; published online 5 October 2016
\end{abstract}

\section{INTRODUCTION}

Major depressive disorder (MDD) is a common and debilitating illness characterized by depressed mood and reduced energy. Accumulating evidence suggests that cerebral metabolic abnormalities may be present regionally (Baxter et al, 1989) and/or globally (Ketter et al, 1999; Kumar et al, 1993) in MDD and that these abnormalities may normalize following treatment with antidepressants (Drevets et al, 2002; Mayberg et al, 2000). Abnormalities in glucose uptake and $\mathrm{rCBF}$ observed in MDD could result from a reduced capacity to utilize glucose, as would be seen in mitochondrial dysfunction, with resulting lower bioenergetic metabolite levels, or from a state of lowered neuronal function resulting in reduced energetic demand on otherwise freely available high-energy phosphate-containing metabolites.

Nucleoside triphosphates (NTP), including adenosine triphosphate (ATP), are generated in mitochondria via oxidative phosphorylation using energy derived from

* Correspondence: Dr DG Harper, Department of Psychiatry, McLean Hospital and Harvard Medical School, 115 Mill Street, Belmont, MA 02478 USA,Tel: +| 617855 3160, Fax: +| 6178553246 , E-mail: dharper@mclean.harvard.edu

Received 5 April 2016; revised II August 2016; accepted 27 August 2016; accepted article preview online 2 September 2016 pyruvate, a product of glucose metabolism. Energy for metabolic activity is released by ATP hydrolysis generating adenosine diphosphate and inorganic phosphate $(\mathrm{Pi})$. ATP can also transfer a high-energy phosphate to phosphocreatine $(\mathrm{PCr})$ and receive it back through the reversible creatine kinase reaction (Erecinska and Silver, 1989). PCr, therefore, serves as a temporal and spatial buffer of high-energy phosphate for use under conditions of high energetic demand and/or at sites distal to mitochondria (Meyer et al, 1984). Pi, in addition to being a metabolic byproduct of ATP utilization, also serves a regulatory function of promoting oxidative phosphorylation as concentration of $\mathrm{Pi}$ builds (Balaban, 2009; Bose et al, 2003; Chance et al, 1986; Schmitz et al, 2012).

Quantifying these energetic metabolites in the brain, therefore, can provide insight into the mechanism of abnormal metabolic activity in MDD. Phosphorus magnetic resonance spectroscopy (31P MRS) allows for the in vivo quantification of three energetic compounds in the brain; NTP; PCr; and Pi; providing a picture of cellular energetic capacity. Previous 31P MRS studies have quantified decreases in NTP in the basal ganglia (Moore et al, 1997; Renshaw et al, 2001), and in the frontal lobes of patients with major depression (Volz et al, 1998). Reductions in PCr in frontal regions have also been observed in severe, as compared with mild, depression (Kato et al, 1992). 
Reductions in NTP (Renshaw et al, 2001) and increased PCr (Iosifescu et al, 2008) have also been associated with likelihood of response to antidepressant treatments in MDD.

Differences in the observation of elevated $v s$ reduced $\mathrm{PCr}$ in depression need to be clarified. One possible explanation is the different tissue composition of samples derived from studies showing reduction of PCr (Kato et al, 1992) vs increased PCr (Iosifescu et al, 2008). Gray matter and white matter have very different energetic demands with gray matter consuming 2-4 times the energy per unit time compared with white matter (Erecinska and Silver, 1989). This factor could partially explain discrepant findings in $\mathrm{PCr}$ as different sampling regions likely have different tissue compositions. 3-dimensional chemical shift imaging (3DCSI) allows for quantification of energetic metabolites in a three-dimensional matrix of voxels over the whole brain allowing for the use of segmentation information to derive gray matter and white matter metabolite estimates (Forester et al, 2009; Hetherington et al, 2001; Mason et al, 1998).

We performed a 3D-CSI 31P MRS study measuring the energetic metabolites $\beta$-NTP (the $\beta$-phosphate is the unique signature of NTP), PCr, and Pi at a 4-Tesla magnetic field strength comparing adult MDD subjects with healthy volunteers. We hypothesized, based on our previous data (Forester et al, 2009), that differences in bioenergetic metabolites between depressed and normal subjects would be tissue class specific (white $v s$ gray matter). We further hypothesized a reduction in $\beta$-NTP (which in the brain largely represents ATP), and a compensatory increase in $\mathrm{PCr}$ in adult MDD subjects, consistent with our previous results (Iosifescu et al, 2008). Moreover, as an exploratory hypothesis, we predicted that Pi would differ between depressed and normal subjects, and the direction of association would be opposite $\beta$-NTP. Finally, for metabolites where we observed significant differences, we explored in MDD subjects the association between metabolite levels and depression symptom severity (the 17-item Hamilton Rating Scale for Depression (HAM-D-17) (Hamilton, 1967) score) or the depression subscores of the Symptom Questionnaire (Kellner, 1987).

\section{MATERIALS AND METHODS}

\section{Subjects}

All subjects signed written informed consent approved by the Institutional Review Boards of Massachusetts General Hospital (MGH) and McLean Hospital before any study procedures were performed.

MDD participants. About 50 subjects with current MDD between ages of 21 and 62 were recruited at the MGH Depression Clinical and Research Program through advertisements and clinical referrals. All subjects met MDD criteria diagnosed by physician-administered Structured Clinical Interview for Axis I Disorders-Patient Edition (SCID-I/P) (First et al, 1996) and had a score of $\geqslant 16$ on the Ham-D-17 at the screen visit. The exclusion criteria for this study were: women lactating or pregnant, or women of childbearing potential not using a medically accepted means of contraception, serious suicidal risk, serious or unstable medical illness, seizure disorder, history of mental disorders secondary to general medical conditions, substance use disorders, including alcohol, active within the last 12 months, any psychotic disorder, bipolar disorder, antisocial personality disorder, concurrent use of psychotropic drugs, hypothyroidism, subjects with existing diagnoses of dementia or with scores $\leqslant 27$ on the Folstein Mini Mental Status Examination, history of non response in the current major depressive episode to more than one adequate antidepressant trial (defined as 6 weeks or more of treatment with citalopram $40 \mathrm{mg} / \mathrm{day}$, or its antidepressant equivalent), history of electroconvulsive therapy in the last 6 months, history of intolerance to citalopram or escitalopram, and subjects with any contraindications to MRI, including metallic implants or severe claustrophobia. If a subject had been recently treated with antidepressants, a medication-free period of at least 2 weeks (4 weeks for fluoxetine) was required before scanning.

Healthy volunteers. We also recruited 30 healthy volunteers between the ages of 21 and 62 using advertisements in local media. All healthy subjects were unmedicated and had no lifetime history of major neurological, medical, psychiatric disorder, or head injury by self-report. Healthy volunteers underwent the physician-administered Structured Clinical Interview for DSM-III-R Axis I Disorders-Patient Edition (SCID-P) (Spitzer et al, 1992) to rule out any Axis I psychopathology.

\section{Procedures}

Approximately 2 weeks after the screening visit (range 1-3 weeks), eligible participants were scheduled for 31P MRS data acquisition at the Brain Imaging Center at McLean Hospital. Eligible MDD subjects had a repeat (baseline) visit at MGH within $24 \mathrm{~h}$ post MRS data acquisition, when HamD-17 was re-administered along with the Symptom Questionnaire (Kellner, 1987), a 92-item self-rating instrument with scales for depression, anxiety, somatization, and anger, as well as contentment, friendliness, relaxation, and somatic well-being.

31P MRS acquisition, processing, and analysis. A dualtuned proton-phosphorus TEM head coil (Bioengineering, Minneapolis, MN) operating at $170.3 \mathrm{MHz}$ for proton and $68.95 \mathrm{MHz}$ for phosphorus was used for all anatomical imaging and spectroscopy. High-contrast, $\mathrm{T}_{1}$-weighted sagittal and axial image sets (TE/TR $=6.2 / 11.4 \mathrm{~ms}$, field-of-view $(\mathrm{FOV})=22 \times 22 \mathrm{~cm}$, readout duration $=4 \mathrm{~ms}$, receive bandwidth $= \pm 32 \mathrm{kHz}$, in-plane matrix size $=128 \times 256$ (sagittal), $256 \times 256$ (axial), in-plane resolution $=1.90 \times 0.94 \mathrm{~mm}$ (sagittal), $\quad 0.94 \times 0.94 \mathrm{~mm}$ (axial), axial-plane matrix size $=32$ (sagittal), 64 (axial) axial-plane resolution $=2.5 \mathrm{~mm}$ (sagittal and axial), scan time $=2 \mathrm{~min}, 30 \mathrm{~s}$ (sagittal), $5 \mathrm{~min}$ (axial)) of the entire brain were acquired using a three-dimensional-, magnetization-prepared FLASH imaging sequence (3D-mpFLASH).

Phosphorus ${ }^{31} \mathrm{P}$ 3D-CSI was done using the following acquisition parameters: $\mathrm{TR}=500 \mathrm{~ms}$; tip-angle $=32$ degrees; $\mathrm{Rx}$ bandwidth $= \pm 2 \mathrm{kHz}$; complex-points $=1024$; readout duration $=256 \mathrm{~ms}$; pre-pulses $=10$; pre-acquisition delay $=1.905 \mathrm{~ms} ; \quad \mathrm{FOV}$ $(\mathrm{x}, \mathrm{y}, \mathrm{z})=330 \mathrm{~mm}$; nominal volume $=13.1 \mathrm{cc} ;$ maximum phase- 
encode matrix dimension $(\mathrm{x}, \mathrm{y}, \mathrm{z}) 14 \times 14 \times 14$ (zero-filled out to $16 \times 16 \times 16$ prior to reconstruction) as described in our prior publications (Forester et al, 2009; Harper et al, 2014).

${ }^{31} \mathrm{P}-\mathrm{MRS}$ processing and analysis. Briefly, the spatially resolved, ${ }^{31} \mathrm{P} 3 \mathrm{D}$-CSI grid was co-registered with the axial T1-weighted images such that the grid was centered midsagittally inside the brain according to anatomical landmarks in both the sagittal and axial planes and a $4 \times 7 \times 3$ matrix of voxels was centered within the brain as described in our prior publications (Forester et al, 2009; Harper et al, 2014).

All offline image processing used commercial and customwritten software for the purpose of tissue segmentation, partial volume analysis and grid-shifting. For ${ }^{31} \mathrm{P} 3 \mathrm{D}-\mathrm{CSI}$ spectral fitting, we used a spectral time-domain fitting program, based on the Marquardt-Levenberg non-linear, least squares algorithm, incorporating prior knowledge of spectral peak assignments, chemical-shifts, and J-coupling constants as described in our prior publications (Forester et al, 2009; Harper et al, 2014; Jensen et al, 2002). Representative data are shown in Figure 1.

Tissue segmentation and image post processing. Using the FSL Brain Extraction Tool (FMRIB) (Smith, 2002), the extracted brain images were then segmented into different classes, white matter, gray matter, and CSF, using FMRIB's Automated Segmentation Tool (FAST) (FMRIB) (Zhang et al, 2001). Results from this automated segmentation process were then used to determine the contributions of tissue type (gray or white matter) and cerebrospinal fluid in each MRS voxel. In this process, we convolved the mathematically modeled, three-dimensional point-spread function (3D-PSF) from the sparse k-space sampling scheme, digitally sampled in a $256 \times 256 \times 64$ matrix, with the co-registered binary images (also digital matrices of $256 \times 256 \times 64)$ to obtain theoretically correct pixel counts of the contribution of each tissue type to each voxel based on the 3D-PSF weighted distribution (Hetherington et al, 2001; Jensen et al, 2002; Mason et al, 1998).

\section{Statistics}

Primary hypothesis. Comparison of metabolite levels between subjects with major depression and controls was performed as described previously (Forester et al, 2009). A separate linear mixed effects model was constructed for each of the three metabolites measured ( $\beta$-NTP, PCr, and $\mathrm{Pi}$ ) in brain. A minimal model first identified individual voxel peaks that were questionable due to contamination by muscle or other artifact. Minimal models included a random effect of subject and a fixed effect of total phosphorus signal for normalization. Voxels were rejected if the studentized residual from the minimal model for each voxel was $>3$ or $<-3$. The full model used for data analysis included a random effect of subject and fixed effects of condition (depressed or normal), partial volume (expressed as percent tissue), gray-white matter difference (expressed as a real number from -100 to 100$)$, and the sum of the total phosphorus signal to normalize the dependent variable. Age and sex were included as control covariates.

Because of our expectation that associations of diagnosis with metabolite concentration could vary by tissue content, we tested first for an interaction between tissue type and diagnosis. We tested for the following: (1) associations of the differences between gray matter and white matter metabolite concentrations with depression (significance of the gray-

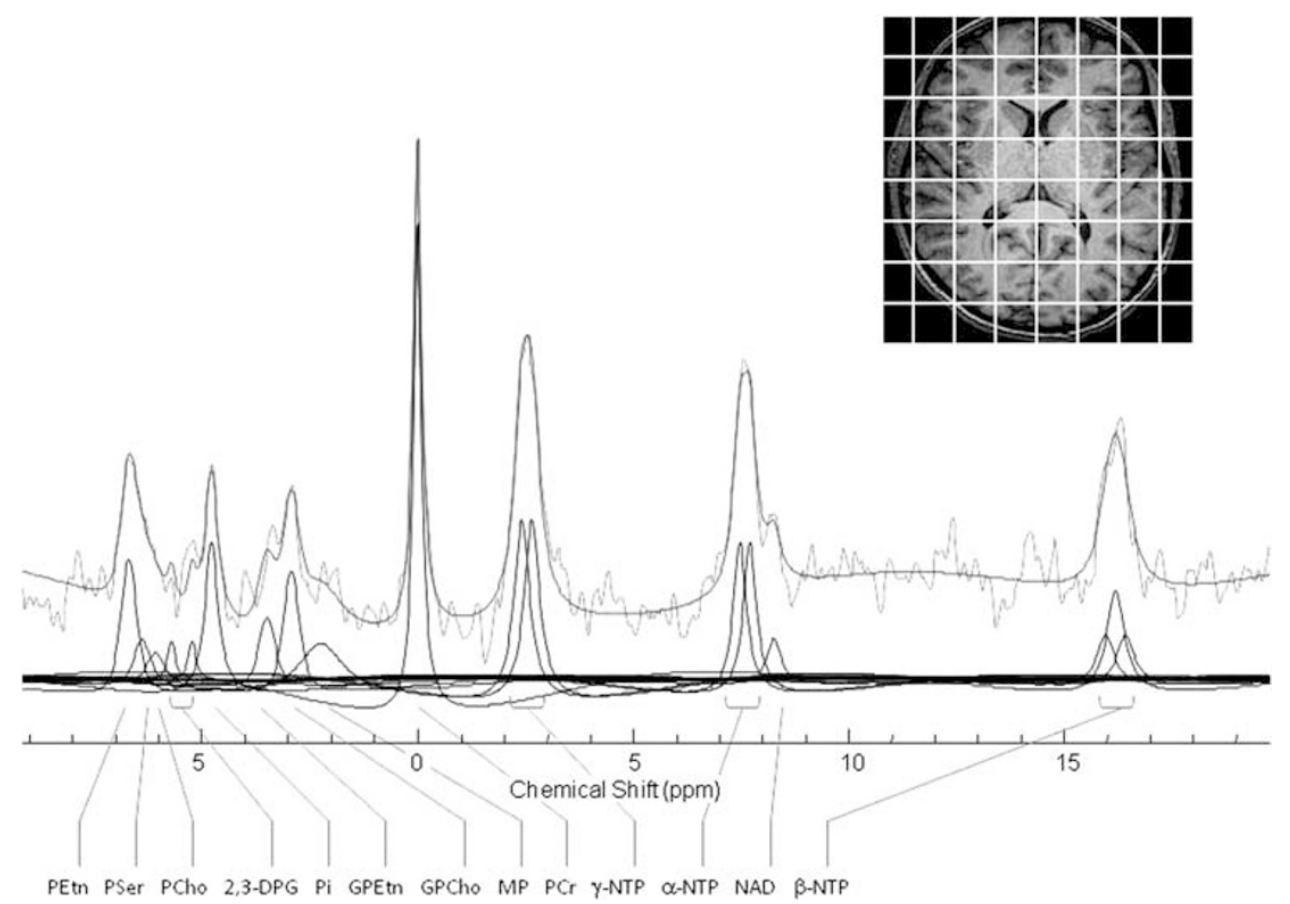

Figure I 3IP spectrum from a single voxel displayed with modeled fit and spectral sub-components. Spectrum is filtered with $12 \mathrm{~Hz}$ exponential filtering for display. 2,3 DPG, diphosphoglycerine; GPCho, glycerphosphocholine; GPEtn, glycerophosphoethanolamine; MP, membrane phospholipids; NAD, dinucleotides; $\alpha-, \beta-, \gamma$-NTP, adenosine triphosphate; PCho, phosphocholine; PCr, phosphocreatine; PEtn, phosphoethanolamine; Pi, inorganic phosphate; PSer, phosphoserine. 
white matter difference by condition interaction), and (2) when this higher order interaction was not significant, we tested for associations in total tissue, which we defined as the mean difference in the metabolite of interest in a voxel composed of equal amounts of gray matter and white matter. To aid in interpretation, we estimated mean metabolite contributions from gray matter and white matter in voxels of mixed composition as previously described (Forester et al, 2009). Bonferroni corrections were applied for the energetic compounds (PCr: $\beta$-NTP) in our primary hypothesis to account for our choice of two outcomes. Accordingly, the $p$-value was multiplied by 2 and the resulting value required for significance remained at 0.05 . Because $\mathrm{Pi}$ was an exploratory outcome, corresponding $p$-values were left uncorrected. Linear models were fitted with the restricted maximum likelihood (REML) method employed by JMP release 12 (SAS; Cary, NC).

Secondary hypotheses. We next tested whether intensity of symptoms in the depressed group predicted metabolite levels. We used an analogous approach to our test of the primary hypothesis, except, in place of condition, we inserted symptom intensity scores. Therefore, separate linear mixed effects models were constructed for each depressive symptom domain of the Symptom Questionnaire (depression, anxiety, somatic, and anger-hostility) and the HAM-D-17 using metabolites where we had previously observed a significant effect of condition (Dmitrienko et al, 2003). We tested for the following: (1) associations of the differences between gray matter and white matter metabolite concentrations with SQ domain or HAM-D-17 total score (significance of the gray-white matter difference by symptom test interaction), and (2) when these higher order interactions were non-significant, we tested for associations in total tissue as defined above. Bonferroni corrections were applied for the total number of symptom dimensions (5) multiplied by the number of metabolites passing the gatekeeper test (2) (Dmitrienko et al, 2003). Therefore, the $p$-value was multiplied by 10 . Statistical significance required a corrected $p$-value of 0.05 corresponding to an uncorrected $p$-value of 0.005 .

Table I Demographic Characteristics of the Sample Including Age and Sex

\begin{tabular}{ccccc}
\hline & Normal & Depressed & Test statistic & p-value \\
\hline Age & $39.03 \pm$ 12.46 & $43.36 \pm 13.62$ & $t(78)=1.42$ & $p=0.16$ \\
& $($ mean \pm SD $)$ & $($ mean \pm SD $)$ & & \\
Sex & 17M: 13F & $27 \mathrm{M}: 23 \mathrm{~F}$ & $\chi^{2}=0.05 ;$ & $p=0.80$ \\
\hline
\end{tabular}

\section{RESULTS}

Demographic characteristics of the study population are presented in Table 1. Tissue composition of 31P MRSI is presented in Table 2.

\section{Primary Hypothesis}

PCr showed a significant tissue type by condition interaction $\left(\mathrm{F}_{1,5931}=17.05 ; p<0.001\right)$ indicating that gray matter $\mathrm{PCr}$ tissue content and white matter $\mathrm{PCr}$ tissue content of depressed and healthy comparison subjects varied differently across conditions. Therefore, we investigated the PCr content of gray matter in healthy controls and depressed subjects, and white matter PCr in healthy controls and depressed subjects. Gray matter PCr content was 5\% higher in depressed subjects compared with healthy comparison subjects $\left(t_{278}=-2.37 ; p<0.04\right)$, and white matter $\mathrm{PCr}$ content in depressed patients was $4 \%$ reduced from levels seen in the healthy comparison subjects $\left(t_{127}=2.44 ; p=0.03\right.$; Figure 2).

There was no significant tissue type by condition interaction with $\beta$-NTP $\left(\mathrm{F}_{1,6007}=0.60 ; p=0.9\right)$ indicating no specific tissue-based differences between depressed and healthy controls. We next examined a tissue neutral approach by testing the mean difference of $\beta$-NTP in voxels set to represent equal amounts of gray matter and white matter. Although we observed $\beta$-NTP in the depression group to be $2 \%$ higher than in the control group, this difference was not significant $\left(t_{99}=-1.77 ; p=0.16\right)$.

$\mathrm{Pi}$ did show a significant tissue type by condition interaction $\left(\mathrm{F}_{1,5957}=8.40 ; p=0.004\right)$ indicating that between

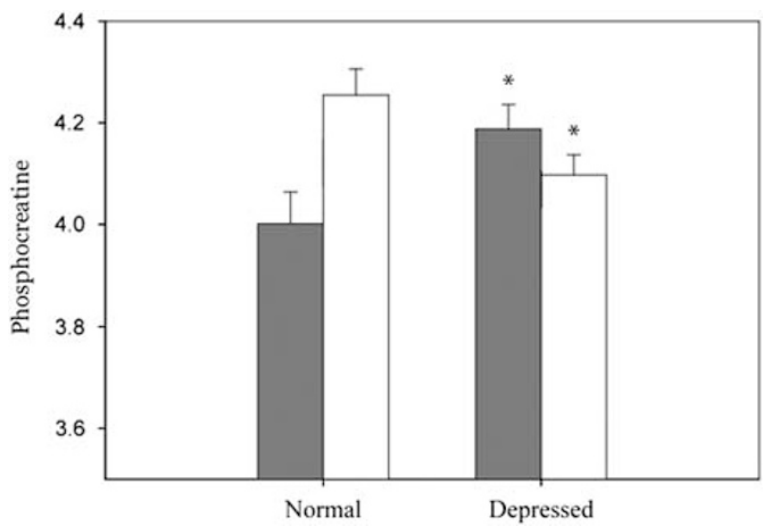

Figure 2 Least squares means ( \pm SE), adjusted for total phosphorus signal in both gray matter and white matter of levels of phosphocreatine in both depressed and normal individuals. ${ }^{*} p<0.05$ when compared with same tissue type of normal subjects. Findings were adjusted for multiple comparisons.

Table 2 Least Squares Mean Partial Volume Expressed as Percent Tissue, and Tissue Type Expressed as a Real Number between - I00 (100\% White Matter) and 100 (100\% Gray Matter) in Normal Controls and Patients with Major Depressive Disorder

\begin{tabular}{|c|c|c|c|c|}
\hline & Normal (LS mean $\pm \mathrm{SE}$ ) & Depressed (LS mean \pm SE) & Test statistic & $p$-value \\
\hline Partial volume & $85.92 \pm 1.10$ & $85.87 \pm 0.85$ & $t(76)=0.03$ & $p=0.98$ \\
\hline Tissue type & $-13.8 \mid \pm 1.17$ & $-|4.10 \pm 0.9|$ & $t(76)=0.20$ & $p=0.84$ \\
\hline
\end{tabular}


depressed and healthy comparison subjects, gray matter and white matter Pi content varied differently. In the case of gray matter, $\mathrm{Pi}$ content was $7 \%$ lower in depressed subjects than what was observed in healthy comparison subjects $\left(t_{573}=2.90\right.$; $p=0.004)$. White matter PCr was not significantly different between healthy comparison subjects and depressed subjects $\left(t_{191}=-0.89 ; p=0.4\right.$; Figure 3$)$.

\section{Secondary Hypotheses}

We next tested whether levels of metabolites, which showed significant findings in the group comparison ( $\mathrm{PCr}$ and $\mathrm{Pi}$ ), had significant interactions between tissue type and HAMD-17 or the self-report Symptom Questionnaire were associated with the metabolite levels in total tissue. The results of these interaction analyses are shown in Table 3.

PCr was found to have a significant interaction between the somatic domain of the SQ and tissue type $(t=3.86$; $p=0.001)$. The somatic scale of SQ includes items related to fatigue (weakness), pain (headaches, body aches), difficulty breathing, tachycardia, and gastro-intestinal complaints (appetite, nausea). Further examination of the individual tissue types (gray matter and white matter) revealed that the somatic domain of the SQ was significantly associated with PCr in gray matter $(t=4.84 ; p=0.0001)$, but did not show any effect in white matter $(t=0.181 ; p=1.0)$ (Figure 4$)$.

$\mathrm{Pi}$ was found to have a significant tissue type interaction with the HAM-D score $(t=3.45 ; p=0.006)$ and with the depression domain of the SQ $(t=4.05 ; p<0.001)$. Gray matter Pi did not show a significant association with HAMD-17 score $\left(t_{446}=2.40 ; p=0.164\right)$, whereas white matter $\mathrm{Pi}$ did show a trend toward a negative association $\left(t_{129}=-2.63 ; p=0.095\right)$. Gray matter $\mathrm{Pi}$ did not show a significant association with the depression domain score of the SQ $(t=2.08 ; p=0.381)$, whereas white matter Pi showed a significant negative association with the depression domain score of the SQ $(t=-3.98 ; p=0.001)$. The depression scale of SQ includes items related to sad mood, anhedonia, guilt,

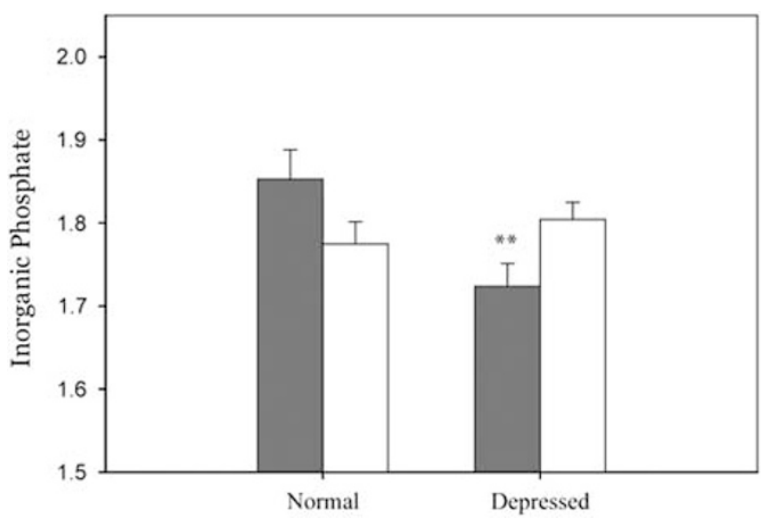

Figure 3 Least squares means $( \pm \mathrm{SE}$ ), adjusted for total phosphorus signal in both gray matter and white matter of levels of phosphocreatine in both depressed and normal individuals. $* * * 0.01$ when compared with same tissue type of normal subjects.

Table 3 Results from Mixed Effects Model of Subjects Intensity of Depression Symptoms and Tissue Type Interaction (W/G Interaction), and Total Tissue Metabolite Concentrations

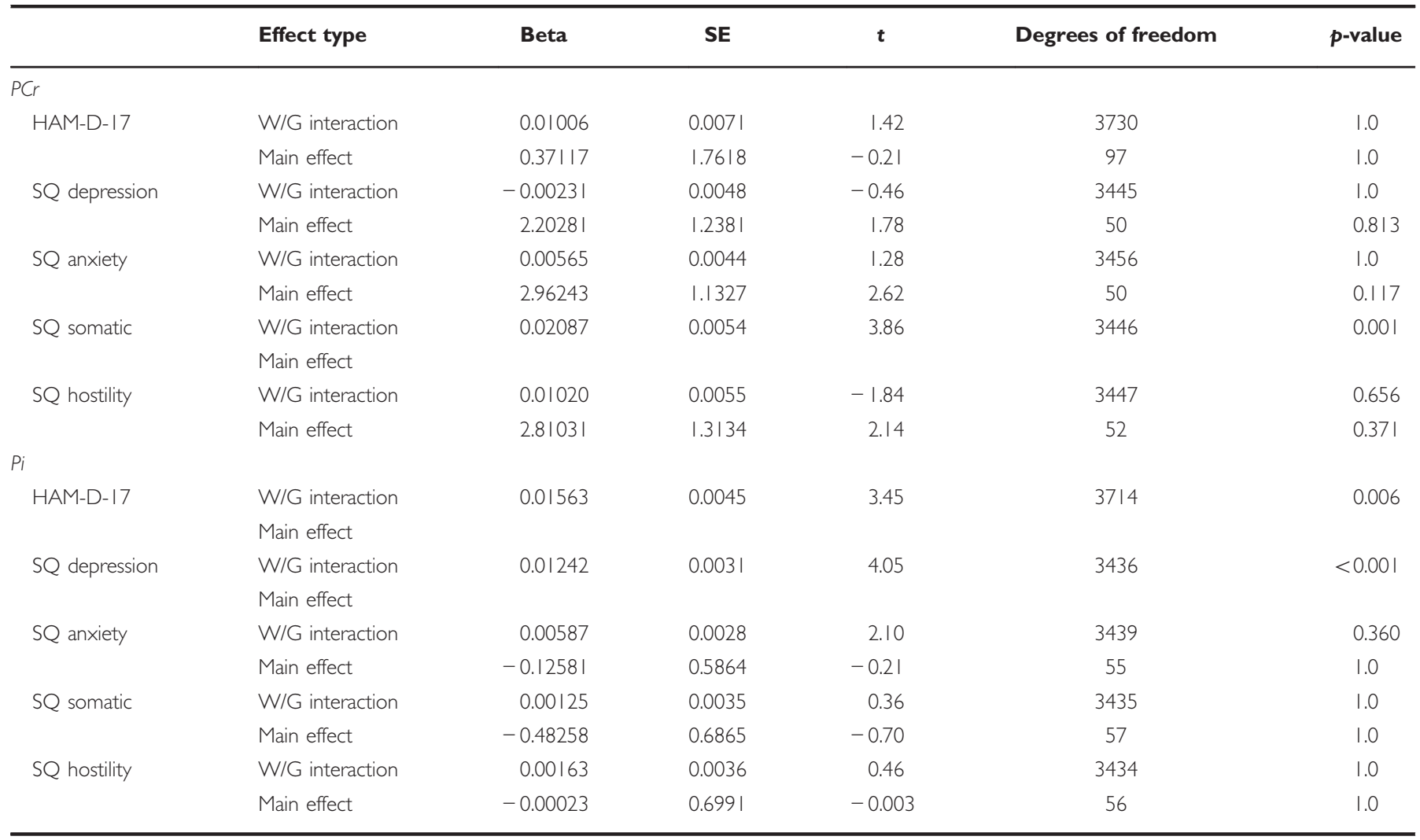

A significant effect in the higher order interaction precluded further testing. Individual effects in each tissue type for significant models are described in the text. 
a

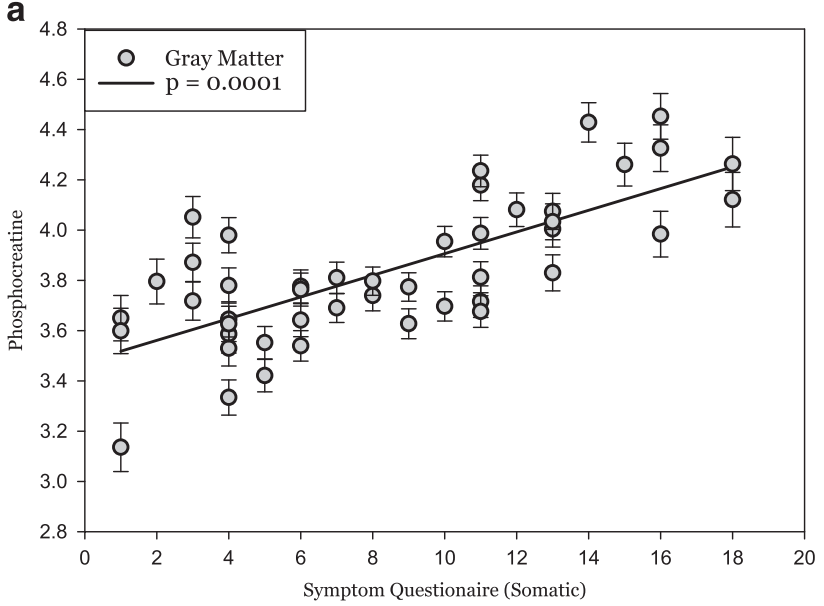

b

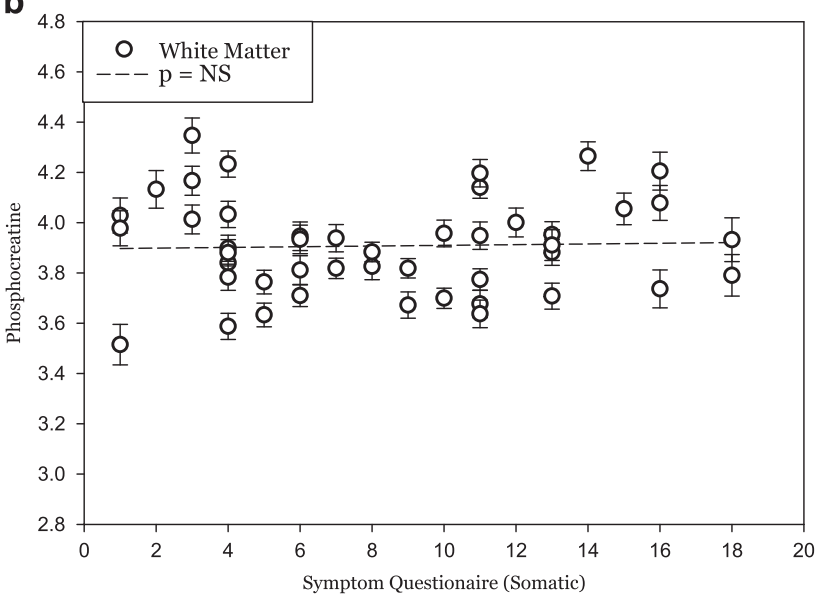

Figure 4 Least squares means plot of individual subjects score on the Symptom Questionnaire, which significantly predicted levels of phosphocreatine (PCr) in (a) gray matter $(t=4.84 ; p=0.000 \mathrm{I})$ but not in (b) white matter $(t=0.181 ; p=1.0)$.

worthlessness (feeling like a failure), hopelessness, and thoughts about death or dying (Figure 5).

\section{DISCUSSION}

In a large sample of unmedicated MDD subjects and healthy volunteers, we found that abnormalities in a key brain bioenergetic metabolite (PCr) were tissue-specific (ie, higher in gray matter and lower in white matter) in depressed subjects when compared with controls. This is consistent with previous reports of tissue-specific abnormalities in brain bioenergetic metabolites (Forester et al, 2009). These results may explain the apparent discrepancy in previous reports using non-segmented brain voxels, showing either reduced (Kato et al, 1992) or increased (Iosifescu et al, 2008) $\mathrm{PCr}$ in MDD.

Both the synthesis and utilization of ATP are vital processes for neuronal activity and survival (Erecinska and Silver, 1989; Lehninger, 2000). However, ATP is only stored in relatively low concentrations even in cells with high- and fluctuating energy requirements (Infante et al, 1965). Such concentrations alone are able to fuel only a brief period of activity before complete ATP depletion. Fortunately, cells are able to draw upon large quantities of intracellular highenergy phosphate from $\mathrm{PCr}$ to continuously and efficiently replete ATP through the creatine kinase reaction (Clarke and Sokoloff, 1999; Iyengar, 1984; Kenyon and Reed, 1983; Wallimann et al, 1992; Wyss and Kaddurah-Daouk, 2000).

Our finding of increased $\mathrm{PCr}$ in the gray matter of MDD subjects may reflect decreased metabolic processes and, therefore, lower utilization of ATP and ultimately accumulation of high-energy phosphate in PCr. A second possible explanation for increased $\mathrm{PCr}$ in gray matter would be increased mitochondrial oxidative phosphorylation in the context of unchanged or even increased metabolic demand. $\mathrm{PCr}$ acts as a reservoir of high-energy phosphate in tissues with high metabolic demand like the brain. Metabolic studies utilizing FDG-PET and SPECT have shown both increases and decreases in glucose metabolism in the brains of patients with MDD (Baxter et al, 1989; Drevets et al, 1992; Mayberg et al, 1994) and fMRI studies show a similar bidirectional pattern (Diener et al, 2012). In an exploratory analysis gray matter $\mathrm{PCr}$ was increased in patients reporting higher scores on the somatization subscale of the SQ (primarily representing increased physical fatigue, aches, and pains). This is consistent with the previous studies where subjects with atypical depression (also associated with excessive fatigue) were the ones that exhibited highest values of $\mathrm{PCr}$ at baseline and responded best to treatment with thyroid hormones (Iosifescu et al, 2008).

The gray matter localization of our findings is intriguing. Post-mortem brain studies have consistently shown that the most important cellular abnormality in MDD is represented by reductions in glial cells and specifically in astrocytes, rather than neurons (Cobb et al, 2016; Ongur et al, 1998; Rajkowska and Stockmeier, 2013). Astrocytes (and especially protoplasmic astrocytes present in the gray matter) have a crucial role in regulating several energy-intensive processes involving neurons, including glucose metabolism, neurotransmitter uptake (particularly glutamate and the cycling from glutamate-glutamine), synaptic development and maturation, and the blood-brain barrier (Kettenmann and Ranson, 2005). Therefore, the PCr changes in gray matter of MDD subjects may be related to specific abnormalities in astrocyte bioenergetic metabolism.

In contrast to our hypothesis, we did not find significant differences in $\beta$-NTP (which in the brain approximates ATP levels) between MDD subjects and healthy volunteers. This may be related to the spectroscopic imaging method we used and to the time resolution of changes in ATP and PCr levels. 31P MRS measures overall concentrations of metabolites during the time of MRS acquisition and is insensitive to rapid changes in metabolite concentrations. Our results primarily demonstrate imbalance of $\mathrm{PCr}$, the longer-term storage of energy-rich phosphates.

$\mathrm{Pi}$, the 'low energy' metabolite of ATP, was observed to be lower in gray matter in depressed subjects when compared with normal controls. This may suggest, based on Pi's role in the stimulation of oxidative phosphorylation, that there is not increased pressure on mitochondrial oxidative phosphorylation to produce ATP and consequently PCr. Such an increase in oxidative phosphorylation might be expected given all the neuroplastic processes involved in the normal brain response 

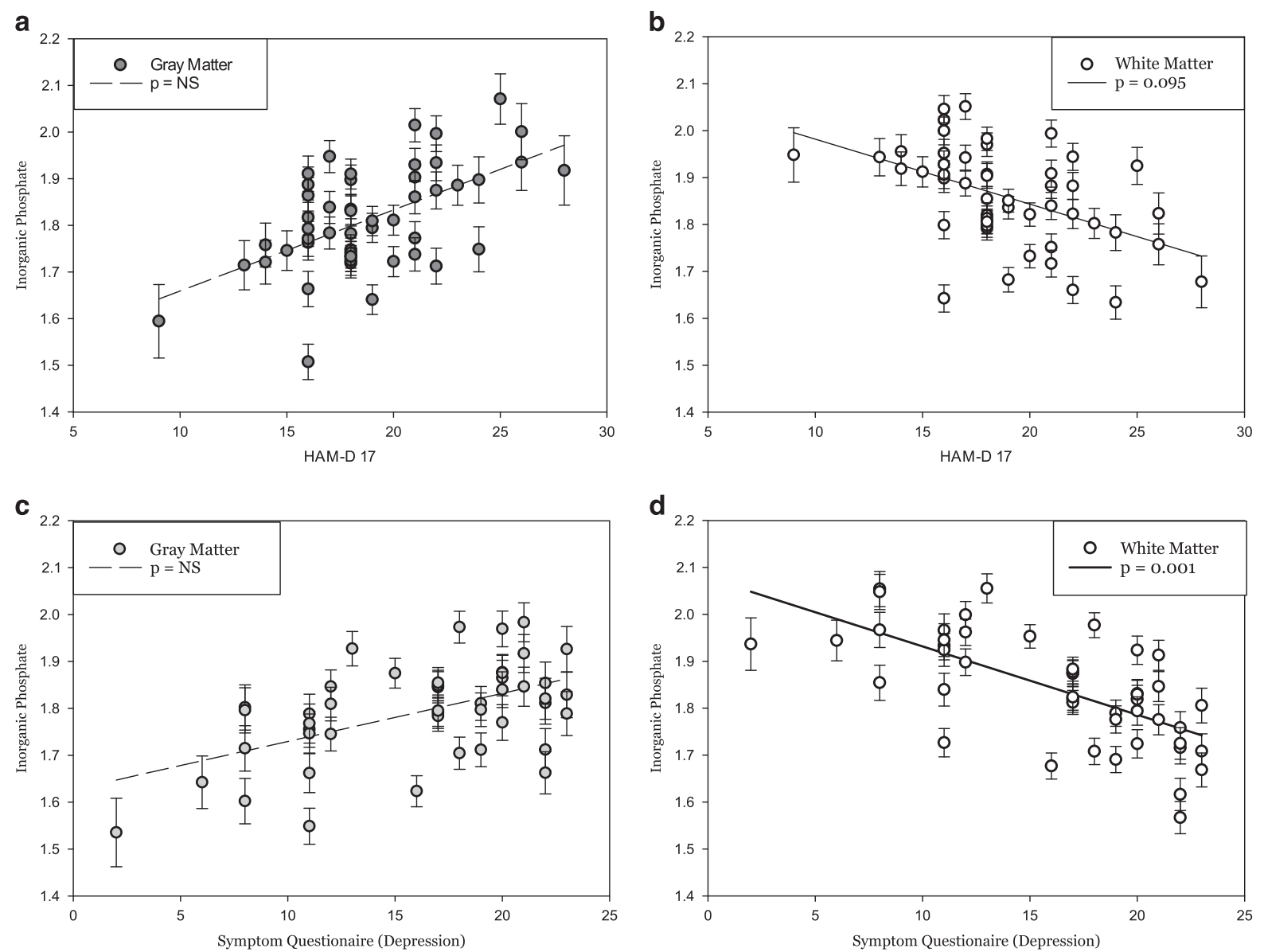

Figure 5 Least squares means plot of individual subjects (a-b) HAM-D scores and scores on the (c-d) Symptom Questionnaire (depression), which significantly predicted levels of gray matter and white matter inorganic phosphate (Pi) in depressed subjects. (a) Gray matter Pi showed no association to HAMD- 17 score $\left(t_{446}=2.40 ; p=0.164\right)$, and (b) white matter Pi showed a significant negative association $\left(t_{129}=-2.63 ; p=0.095\right)$. (c) Analogous to the results from the HAM-D-17, gray matter Pi also showed no association with the SQ (depression) score $\left(t_{339}=2.08 ; p=0.381\right)$, whereas $(d)$ white matter $\mathrm{Pi}$ showed a highly significant association to the SQ (depression) score $\left(t_{120}=-3.98 ; p=0.00 \mathrm{I}\right)$.

to stress (Jeanneteau and Arango-Lievano, 2016). Instead our results support the idea that metabolic processes in gray matter in major depression have slowed, resulting in accumulation of high-energy phosphates in PCr.

We also observed a severity dependent change in $\mathrm{Pi}$, contrasting by tissue class. The results were numerically consistent, regardless whether depression severity was measured with HAM-D or SQ depression: more severe depression was associated with higher Pi levels in the gray matter (but only the association with HAM-D was a statistical trend) and with lower Pi levels in the white matter (but only the association with SQ depression was significant). The higher Pi in the white matter of severely depressed subjects was consistent with the overall direction of the contrast between MDD and control subjects. However, the Pi association with depression severity in the gray matter was opposite of what we would have expected to see if the state of depressive illness was driving the overall lowered gray matter Pi in the depression group. Therefore, although the depression group overall showed a decrease in gray matter $\mathrm{Pi}$, and subsequent activation of oxidative phosphorylation in gray matter, those with more severe depression had a relatively greater Pi sending an increased signal to stimulate oxidative phosphorylation.
Overall, glucose metabolism, measured with FDG-PET, has been highly variable across studies in patients with major depression. One of the differences hypothesized to account for this variability is severity of depression (Little et al, 2005). Hypermetabolism in depressed individuals in previous studies may be linked to more severe symptoms (Mayberg et al, 1997), whereas hypometabolism appears linked to less severe illness (Baxter et al, 1989; Brody et al, 1999; Little et al, 2005; Milak et al, 2009). Our findings support the hypothesis that hypermetabolism could be a consequence of depression severity, as we observed Pi to be increased in gray matter, thereby stimulating oxidative phosphorylation, based on severity of depression.

To our knowledge, this cohort represents the largest sample of MDD subjects scanned with 31P MRS, and our MRS methods include high-definition MRSI at $4 \mathrm{~T}$. The most significant study limitation is related to the 'static' nature of 31P MRS, which measures steady-state concentrations and is unable to provide measures of dynamic change in bioenergetic metabolites. The metabolite changes reported here represent a novel state of metabolic equilibrium in MDD subjects; the levels themselves do not explain whether oxidative phosphorylation has increased, decreased, or stayed the same. Additional studies utilizing dynamic 
methods such as magnetization-transfer MRS will be able to refine our current results providing dynamic measures of the chemical exchange process between $\mathrm{PCr}$ and ATP $(\mathrm{PCr} \rightarrow$ ATP) catalyzed by creatine kinase, by measuring the flux of the phosphate exchange reaction between PCr and ATP.

A second limitation results from the cross-sectional nature of the study design, which does not allow us to speculate on the issue of state $v s$ trait of availability of high-energy phosphates beyond severity dependence. Previous work (Iosifescu et al, 2008) suggests that the high-energy phosphate balances are impacted by antidepressant medication. Further studies following effective treatment will allow for the elaboration of these issues.

A further limitation inherent in our methodology is the ever-present yet slight phosphorus signal contamination in our brain voxels arising from skeletal muscle (primarily the temporalis muscle) where phosphorus concentrations are $\sim 10$-fold higher than in brain. Although we attempted to minimize this contamination by staying a nominal voxel's width away from the skull boundary, the nature of the PSF arising from Fourier reconstruction of our voxels will always result in cross-voxel signal bleed, which could potentially affect our results. Further refinements in our methods will seek to maximize the amount of cortical gray matter included in our analysis while minimizing signal contribution from skeletal muscle to an acceptable level.

Finally, the global measures of phosphorus metabolites we collected in this study can obscure regional effects. Although we have been able to overcome difficulties in interpreting differences in tissue type with the use of 3D-CSI and mixed effects models, it is at the cost of regional specificity that would be available in a single voxel study. We are also unable to detect correlations between brain bioenergetic abnormalities and any systemic changes in metabolism, expressed either as daily exercise, oxidative metabolism demand, or exercise capacity. Future studies will be needed to elucidate correlations between bioenergetic changes in the brain and the rest of the body.

Despite these limitations, our study adds to a body of literature supporting a model of bioenergetic dysfunction in subjects with major depression. Our data further clarifies previous results by defining tissue-specific abnormalities in brain bioenergetics in MDD. These results have theoretical and practical implications, enhancing our understanding of the pathology of MDD and supporting methods, which enhance brain cellular bioenergetics as potential antidepressant treatments (Nierenberg et al, 2013).

\section{FUNDING AND DISCLOSURE}

This work was supported by the National Institute of Mental Health (NIMH) grant K23MH067111 (Dr Iosifescu) and the Roger's Family Foundation (Dr Harper). Dr Harper has received research support through McLean Hospital from Eli Lilly, Merck, Navidia, and AstraZeneca, and he serves as a consultant to Abide Therapeutics and Eisai. Dr Jensen and Dr Ravichandran report no conflicts of interest. Dr Perlis has served on scientific advisory boards or consulted to Genomind, Healthrageous, Perfect Health, Psybrain, and RID Ventures. He receives patent fees from UBC. Dr Fava has received research support from Abbott Laboratories, Alkermes, American Cyanamid-Aspect Medical Systems,
AstraZeneca, Avanir, BioResearch, BrainCells, Bristol-Myers Squibb, CeNeRx BioPharma, Cephalon, Cerecor, Clintara, Covance, Covidien, Eli Lilly, EnVivo, Euthymics, Forest Pharmaceuticals, FORUM, Ganeden, GlaxoSmithKline, Harvard Clinical Research Institute, Hoffman-LaRoche, Icon Clinical Research, i3 Innovus/Ingenix, Janssen R\&D, Jed Foundation, Johnson \& Johnson Pharmaceutical Research and Development, Lichtwer Pharma, Lorex, Lundbeck, MedAvante, Methylation Sciences, NARSAD, NCCAM, NiiCM, NIDA, NIMH, Neuralstem, Novartis AG, Organon, PamLab, PharmaciaUpjohn, Pharmaceutical Research Associates, Pharmavite, PharmoRx Therapeutics, Photothera, Reckitt Benckiser, Roche, RCT Logic, Sanofi-Aventis US, Shire, Solvay Pharmaceuticals, SMRI, Synthelabo, Takeda, Tal Medical, and Wyeth-Ayerst Laboratories. Dr Fava has been on the advisory board or a consultant for Abbott Laboratories, Acadia, Affectis Pharmaceuticals, Alkermes, Amarin Pharma, Aspect Medical Systems, AstraZeneca, Auspex, Avanir, AXSOME, Bayer AG, Best Practice Project Management, Biogen, BioMarin, Biovail, BrainCells, Bristol-Myers Squibb, CeNeRx BioPharma, Cephalon, Cerecor, CNS Response, Compellis, Cypress Pharmaceutical, DiagnoSearch Life Sciences, Dinippon Sumitomo Pharma, Dov Pharmaceuticals, Edgemont Pharmaceuticals, Eisai, Eli Lilly and Company, EnVivo, ePharmaSolutions, Forum Pharmaceuticals, GenOmind, GlaxoSmithKline, Grunenthal GmbH, i3 Innovus/ Ingenis, Intracellular, Janssen Pharmaceutica, Jazz Pharmaceuticals, Johnson \& Johnson Pharmaceutical Research \& Development, Knoll Pharmaceuticals, Labopharm, Lorex, Lundbeck, MedAvante, Merck, MSI Methylation Sciences, Naurex, Nestle Health Sciences, Neuralstem, Neuronetics, NextWave Pharmaceuticals, Novartis AG-Nutrition 21, Orexigen Therapeutics, Organon Pharmaceuticals, Osmotica, Otsuka, Oamlab, Pfizer, PharmaStar, Pharmavite, PharmoRx Therapeutics, Precision Human Biolaboratory, Prexa Pharmaceuticals, Puretech Ventures, PsychoGenics, Psylin Neurosciences, RCT Logic, Rexahn Pharmaceuticals, Ridge Diagnostics, Roche, Sanofi-Aventis US, Sepracor, Servier Laboratories, Schering-Plough, Solvay Pharmaceuticals, Somaxon Pharmaceuticals, Somerset Pharmaceuticals, Sunovion Pharmaceuticals, Supernus Pharmaceuticals, Synthelabo, Taisho Pharmaceutical, Takeda, Tal Medical, Tetragenex, TransForm, Transcept, Vanda Pharmaceuticals, and Vistagen. Dr Fava has spoken or published for Adamed, Advanced Meeting Partners, American Psychiatric Association, American Society of Clinical Psychopharmacology, AstraZeneca, Belvoir Media Group, Boehrigner Ingelheim GmbH, Bristol-Myers Squibb, Cephalon, CME Institute, Physicians Postgraduate Press, Eli Lilly and Company, Forest Pharmaceuticals, GlaxoSmithKline, Imedex, MGH Psychiatry Academy, Primedia, Reed Elsevier, Novartis AG, Organon Pharmaceuticals, Pfizer, PharmaStar, United BioSource, and Wyeth-Ayerst Laboratories. He holds equity in Compellis and PsyBrain. Dr Fava also has patents for Sequential Parallel Comparison Design (SPCD), which are licensed by MGH to Pharmaceutical Product Development, and a patent application for a combination of Ketamine plus Scopalamine in Major Depressive Disorder, licensed by MGH to Biohaven. Dr Fava holds copyright for the MGH Cognitive \& Physical Functioning Questionnaire (CPFQ), Sexual Functioning Inventory (SFI), Antidepressant Treatment Response Questionnaire (ATRQ), Discontinuation-Emergent Signs \& Symptoms (DESS), Symptoms of Depression Questionnaire (SDQ), and SAFER by Lippincott, Williams \& Wilkins, 
Wolkers Kluwer, and World Scientific Publishing Company. Dr Renshaw has been a consultant to Kyowa Hakko Kirin, Tal Medical, and Ridge Diagnostics. In the past 3 years, Dr Iosifescu has consulted for Avanir, Axsome, CNS Response, INSYS Therapeutics, Lundbeck, Otsuka, Servier, and Sunovion and he has received research support through the Icahn School of Medicine at Mount Sinai from Alkermes, AstraZeneca, Brainsway, Euthymics, Neosync, Roche, and Shire.

\section{REFERENCES}

Balaban RS (2009). Domestication of the cardiac mitochondrion for energy conversion. J Mol Cell Cardiol 46: 832-841.

Baxter LR Jr., Schwartz JM, Phelps ME, Mazziotta JC, Guze BH, Selin CE et al (1989). Reduction of prefrontal cortex glucose metabolism common to three types of depression. Arch Gen Psychiatry 46: 243-250.

Bose S, French S, Evans FJ, Joubert F, Balaban RS (2003). Metabolic network control of oxidative phosphorylation: multiple roles of inorganic phosphate. J Biol Chem 278: 39155-39165.

Brody AL, Saxena S, Silverman DH, Alborzian S, Fairbanks LA, Phelps ME et al (1999). Brain metabolic changes in major depressive disorder from pre- to post-treatment with paroxetine. Psychiatry Res 91: 127-139.

Chance B, Leigh JS Jr., Kent J, McCully K, Nioka S, Clark BJ et al (1986). Multiple controls of oxidative metabolism in living tissues as studied by phosphorus magnetic resonance. Proc Natl Acad Sci USA 83: 9458-9462.

Clarke DD, Sokoloff L (1999). Circulation and energy metabolism of the brain. In: Siegal Gea (ed). Basic Neurochemistry. Lippincott, Williams \& Wilkins: Philadelphia, pp 638-669.

Cobb JA, O'Neill K, Milner J, Mahajan GJ, Lawrence TJ, May WL et al (2016). Density of GFAP-immunoreactive astrocytes is decreased in left hippocampi in major depressive disorder. Neuroscience 316: 209-220.

Diener C, Kuehner C, Brusniak W, Ubl B, Wessa M, Flor H (2012). A meta-analysis of neurofunctional imaging studies of emotion and cognition in major depression. Neuroimage 61: 677-685.

Dmitrienko A, Offen WW, Westfall PH (2003). Gatekeeping strategies for clinical trials that do not require all primary effects to be significant. Stat Med 22: 2387-2400.

Drevets WC, Bogers W, Raichle ME (2002). Functional anatomical correlates of antidepressant drug treatment assessed using PET measures of regional glucose metabolism. Eur Neuropsychopharmacol 12: 527-544.

Drevets WC, Videen TO, Price JL, Preskorn SH, Carmichael ST, Raichle ME (1992). A functional anatomical study of unipolar depression. J Neurosci 12: 3628-3641.

Erecinska M, Silver IA (1989). ATP and brain function. J Cereb Blood Flow Metab 9: 2-19.

First MB, Spitzer RL, Gibbon M, Williams JBW (1996). Structured Clinical Interview for DSM-IV Axis I disorders - Patient edition (SCID-I/P). Biometrics Research Department: New York State Psychiatric Institute. Available at: www.scid4.org.

Forester BP, Harper DG, Jensen JE, Ravichandran C, Jordan B, Renshaw PF et al (2009). (31)Phosphorus magnetic resonance spectroscopy study of tissue specific changes in high energy phosphates before and after sertraline treatment of geriatric depression. Int J Geriatr Psychiatry 24: 788-797.

Hamilton M (1967). Development of a rating scale for primary depressive illness. Br J Soc Clin Psychol 6: 278-296.

Harper DG, Jensen JE, Ravichandran C, Sivrioglu Y, Silveri M, Iosifescu DV et al (2014). Tissue-specific differences in brain phosphodiesters in late-life major depression. Am J Geriatr Psychiatry 22: 499-509.
Hetherington HP, Spencer DD, Vaughan JT, Pan JW (2001). Quantitative (31)P spectroscopic imaging of human brain at 4 Tesla: assessment of gray and white matter differences of phosphocreatine and ATP. Magn Reson Med 45: 46-52.

Infante AA, Klaupiks D, Davies RE (1965). Phosphorylcreatine consumption during single-working contractions of isolated muscle. Biochim Biophys Acta 94: 504-515.

Iosifescu DV, Bolo NR, Nierenberg AA, Jensen JE, Fava M, Renshaw PF (2008). Brain bioenergetics and response to triiodothyronine augmentation in major depressive disorder. Biol Psychiatry 63: 1127-1134.

Iyengar MR (1984). Creatine kinase as an intracellular regulator. J Muscle Res Cell Motil 5: 527-534.

Jeanneteau F, Arango-Lievano M (2016). Linking mitochondria to synapses: new insights for stress-related neuropsychiatric disorders. Neural Plast 2016: 3985063.

Jensen JE, Drost DJ, Menon RS, Williamson PC (2002). In vivo brain (31)P-MRS: measuring the phospholipid resonances at 4 Tesla from small voxels. NMR Biomed 15: 338-347.

Kato T, Takahashi S, Shioiri T, Inubushi T (1992). Brain phosphorous metabolism in depressive disorders detected by phosphorus-31 magnetic resonance spectroscopy. J Affect Disord 26: 223-230.

Kellner R (1987). A symptom questionnaire. J Clin Psychiatry 48: 268-274.

Kenyon GL, Reed GH (1983). Creatine kinase: structure-activity relationships. Adv Enzymol Relat Areas Mol Biol 54: 367-426.

Kettenmann H, Ranson BR (2005). Neuroglia. Oxford University Press: New York.

Ketter TA, Kimbrell TA, George MS, Willis MW, Benson BE, Danielson A et al (1999). Baseline cerebral hypermetabolism associated with carbamazepine response, and hypometabolism with nimodipine response in mood disorders. Biol Psychiatry 46: 1364-1374.

Kumar A, Newberg A, Alavi A, Berlin J, Smith R, Reivich M (1993). Regional cerebral glucose metabolism in late-life depression and Alzheimer disease: a preliminary positron emission tomography study. Proc Natl Acad Sci USA 90: 7019-7023.

Lehninger (2000). Principles of Biochemistry. Worth Publishers, Inc.: New York.

Little JT, Ketter TA, Kimbrell TA, Dunn RT, Benson BE, Willis MW et al (2005). Bupropion and venlafaxine responders differ in pretreatment regional cerebral metabolism in unipolar depression. Biol Psychiatry 57: 220-228.

Mason GF, Chu WJ, Vaughan JT, Ponder SL, Twieg DB, Adams D et al (1998). Evaluation of 31P metabolite differences in human cerebral gray and white matter. Magn Reson Med 39: 346-353.

Mayberg HS, Brannan SK, Mahurin RK, Jerabek PA, Brickman JS, Tekell JL et al (1997). Cingulate function in depression: a potential predictor of treatment response. Neuroreport 8: 1057-1061.

Mayberg HS, Brannan SK, Tekell JL, Silva JA, Mahurin RK, McGinnis S et al (2000). Regional metabolic effects of fluoxetine in major depression: serial changes and relationship to clinical response. Biol Psychiatry 48: 830-843.

Mayberg HS, Lewis PJ, Regenold W, Wagner HN Jr. (1994). Paralimbic hypoperfusion in unipolar depression. J Nucl Med 35: 929-934.

Meyer RA, Sweeney HL, Kushmerick MJ (1984). A simple analysis of the "phosphocreatine shuttle". Am J Physiol 246(5 Pt 1): C365-C377.

Milak MS, Parsey RV, Lee L, Oquendo MA, Olvet DM, Eipper F et al (2009). Pretreatment regional brain glucose uptake in the midbrain on PET may predict remission from a major depressive episode after three months of treatment. Psychiatry Res 173: 63-70.

Moore CM, Christensen JD, Lafer B, Fava M, Renshaw PF (1997). Lower levels of nucleoside triphosphate in the basal ganglia of depressed subjects: a phosphorous-31 magnetic resonance spectroscopy study. Am J Psychiatry 154: 116-118. 
Nierenberg AA, Kansky C, Brennan BP, Shelton RC, Perlis R, Iosifescu DV (2013). Mitochondrial modulators for bipolar disorder: a pathophysiologically informed paradigm for new drug development. Aust N Z J Psychiatry 47: 26-42.

Ongur D, Drevets WC, Price JL (1998). Glial reduction in the subgenual prefrontal cortex in mood disorders. Proc Natl Acad Sci USA 95: 13290-13295.

Rajkowska G, Stockmeier CA (2013). Astrocyte pathology in major depressive disorder: insights from human postmortem brain tissue. Curr Drug Targets 14: 1225-1236.

Renshaw PF, Parow AM, Hirashima F, Ke Y, Moore CM, Frederick Bde B et al (2001). Multinuclear magnetic resonance spectroscopy studies of brain purines in major depression. Am J Psychiatry 158: 2048-2055.

Schmitz JP, Jeneson JA, van Oorschot JW, Prompers JJ, Nicolay K, Hilbers PA et al (2012). Prediction of muscle energy states at low metabolic rates requires feedback control of mitochondrial respiratory chain activity by inorganic phosphate. PLoS One 7: e34118.
Smith SM (2002). Fast robust automated brain extraction. Hum Brain Mapp 17: 143-155.

Spitzer RL, Williams JB, Gibbon M, First MB (1992). The structured clinical interview for dsm-iii-r (scid). i: history, rationale, and description. Arch Gen Psychiatry 49: 624-629.

Volz HP, Rzanny R, Riehemann S, May S, Hegewald H, Preussler B et al (1998). 31P magnetic resonance spectroscopy in the frontal lobe of major depressed patients. Eur Arch Psychiatry Clin Neurosci 248: 289-295.

Wallimann T, Wyss M, Brdiczka D, Nicolay K, Eppenberger HM (1992). Intracellular compartmentation, structure and function of creatine kinase isoenzymes in tissues with high and fluctuating energy demands: the 'phosphocreatine circuit' for cellular energy homeostasis. Biochem J 281 (Pt 1): 21-40.

Wyss M, Kaddurah-Daouk R (2000). Creatine and creatinine metabolism. Physiol Rev 80: 1107-1213.

Zhang Y, Brady M, Smith S (2001). Segmentation of brain MR images through a hidden markov random field model and the expectation-maximization algorithm. IEEE Trans Med Imaging 20: $45-57$. 